\title{
Treatment Modalities for Achalasia Cardia: A Case Study and Literature Review
}

\author{
Andee Dzulkarnaen, Zakaria Lee Say, Bob Syed Hassan, \\ Nizam Hashim and Maya Mazuwin \\ Department of Surgery, School of Medical Sciences, Universiti Sains Malaysia, Health Campus, Kelantan, \\ Malaysia
}

Correspondence should be addressed to: Andee Dzulkarnaen; andee@kb.usm.my

Received 14 May 2013; Accepted 24 June 2013; Published 19 August 2013

Academic Editor: Zeynel A. Mungan

Copyright @ 2013 Andee Dzulkarnaen, Zakaria Lee Say, Bob Syed Hassan, Nizam Hashim and Maya Mazuwin. Distributed under Creative Commons CC-BY 3.0

\section{Abstract}

Achalasia cardia is a benign idiopathic disorder caused by progressive neuronal degeneration in the mesenteric plexus of Auerbach, which causing non-relaxing, hypertensive lower esophageal sphincter (LES) and aperistalsis of the esophageal body. We report a 52-year-old lady presented with progressive dysphagia for 4 months, associated with loss of weight. Other than underweight with $\mathrm{BMI}$ of $18 \mathrm{~kg} / \mathrm{m} 2$ and mild tenderness at epigastrium, clinical examinations were unremarkable.

Upper endoscopy revealed dilated esophagus with evidence of fungal esophagitis, and severe gastritis. Esophageal manometry confirmed achalasia cardia with evidence of aperistalsis of esophageal body and failure of relaxation of lower esophageal sphincter (LES) on swallowing. She had undergone pneumatic dilatation for 3 times before significant improvement in symptoms noticed clinically.

Literatures were reviewed to compare current available therapies for achalasia cardia and it is recommended that the patient should undergo laparoscopic myotomy and partial fundoplication (to prevent free reflux of gastric acid into the esophagus) for better remission. Other treatment modalities such as Botulinum toxin injection and pneumatic dilation can offer dysphagia control, but they are temporary and reversible measures. The objective of this case report is to review the current available treatment modalities for the management of achalasia cardia.

Keywords: Achalasia cardia; endoscopic dilatation; Botulinum toxin.

\section{Introduction}

Achalasia is a benign idiopathic disorder caused by progressive neuronal degeneration in the mesenteric plexus of Auerbach, which causing non-relaxing, hypertensive lower esophageal sphincter (LES) and aperistalsis of the esophageal body (John L. Cameron, 2011).
This functional damage is irreversible and the treatment of esophageal achalasia is mainly palliative.

The most recognized clinical features of the disease are progressive dysphagia, regurgitation, chest pain, and weight loss (Vaezi and Richter, 1998). Repeated 
aspiration pneumonia can be one of the clinical presentations. Achalasia is also a risk factor for the development of esophageal carcinoma, with 140-fold increased risk of cancer compared to general population (John L. Cameron, 2011). The aim of therapy in patients with achalasia is to relieve distal obstruction by reducing the pressure gradient across the LES, which can be treated by Botulinum toxin injection, pneumatic dilatation (PD) or surgical myotomy (Vaezi and Richter, 1998).

\section{Case Report}

A 52 years old lady, presented with progressive dysphagia for 4 months. She complained of difficulty in swallowing food since 4 months ago, started with solid food. She described food stucked at the epigastric region, but able to relieved by taking fluid. Gradually until a week prior to admission, she complained of unable to tolerate fluid as well. It was associated with vomiting whereby she vomited several times per day over the last 2 months. She vomited soon after taking meal. The vomitus consisted of undigested food without bile or blood. She also had intermittent epigastric pain, which she described as burning sensation. Besides, she also had loss of appetite and loss of weight (10kg over the last 2 months). Nevertheless, there was no odynophagia, fever, dyspnea, bone pain or jaundice.

She has underlying bronchial asthma, for which she was using Salbutamol inhaler when necessary. There was no significant past surgical history. She is a housewife and non- smoker. Her mother had cervical cancer.

Clinically she was pink, dehydrated, heart rate $80 / \mathrm{min}$ (regular) and blood pressure $130 / 80 \mathrm{mmHg}$. Her weight was $46 \mathrm{~kg}$ and height was $160 \mathrm{~cm}$, with calculated body mass index of $18 \mathrm{~kg} / \mathrm{m}^{2}$. There was no wasting of temporalis muscle and no cervical lymph nodes palpable. There was no leg edema. Abdominal examination was unremarkable except for mild tenderness over the epigastric region. Lungs were clear with no crepitation bilaterally. Other systemic examinations were unremarkable.

Her hemoglobin level was $14.9 \mathrm{~g} / \mathrm{dl}$; white cell count was 6,700. Serum sodium was $144 \mathrm{mmol} / \mathrm{L}$, potassium level was $3.9 \mathrm{mmol} / \mathrm{L}$, urea of $6.9 \mathrm{mmol} / \mathrm{L}$ and serum creatinine of 86umol/L. Serum phosphate was $1 \mathrm{mmol} / \mathrm{L}$ and serum magnesium was $0.8 \mathrm{mmol} / \mathrm{L}$.

Oesophago-gastric-duodeno-scopy (OGDS) revealed residual food particles accumulated at the lower esophagus, with dilated lower esophagus and generalized whitish lesions (Fig. 1). There was also severe gastritis with bile reflux and mild duodenitis. CLO (Campylobacter-like organism) rapid test was negative. Biopsy of the lower esophagus reported as there is scattered yeast like fungal bodies with budding are seen with positive PAS and GMS staining. However, no dysplasia or malignancy cell seen. Esophageal manometry (Fig. 2(a) and (b)) revealed aperistalsis as no contraction of the body of esophagus during swallowing and failure of relaxation of LES on swallowing, which was consistent with achalasia. 


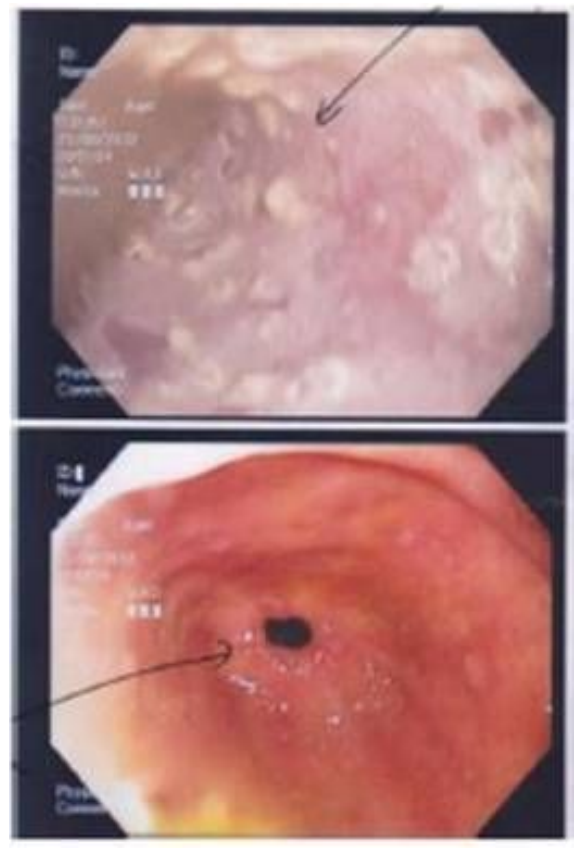

Fig. 1: OGDS Findings with Dilated Esophagus Residual Food Particles Accumulated at the Lower Esophagus, with Dilated Lower Esophagus and Generalized Whitish Lesions (Top) and Severe Gastritis (Bottom)

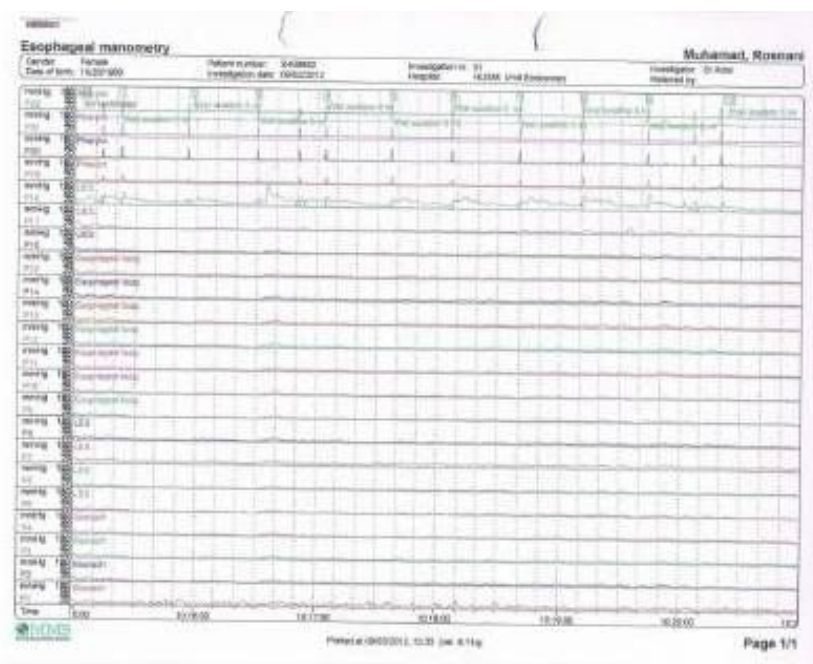

Fig. 2(a): Esophageal Manometry Showing Aperistalsis of the Body of Esophagus and Failure of Relaxation of LES on Swallowing. 


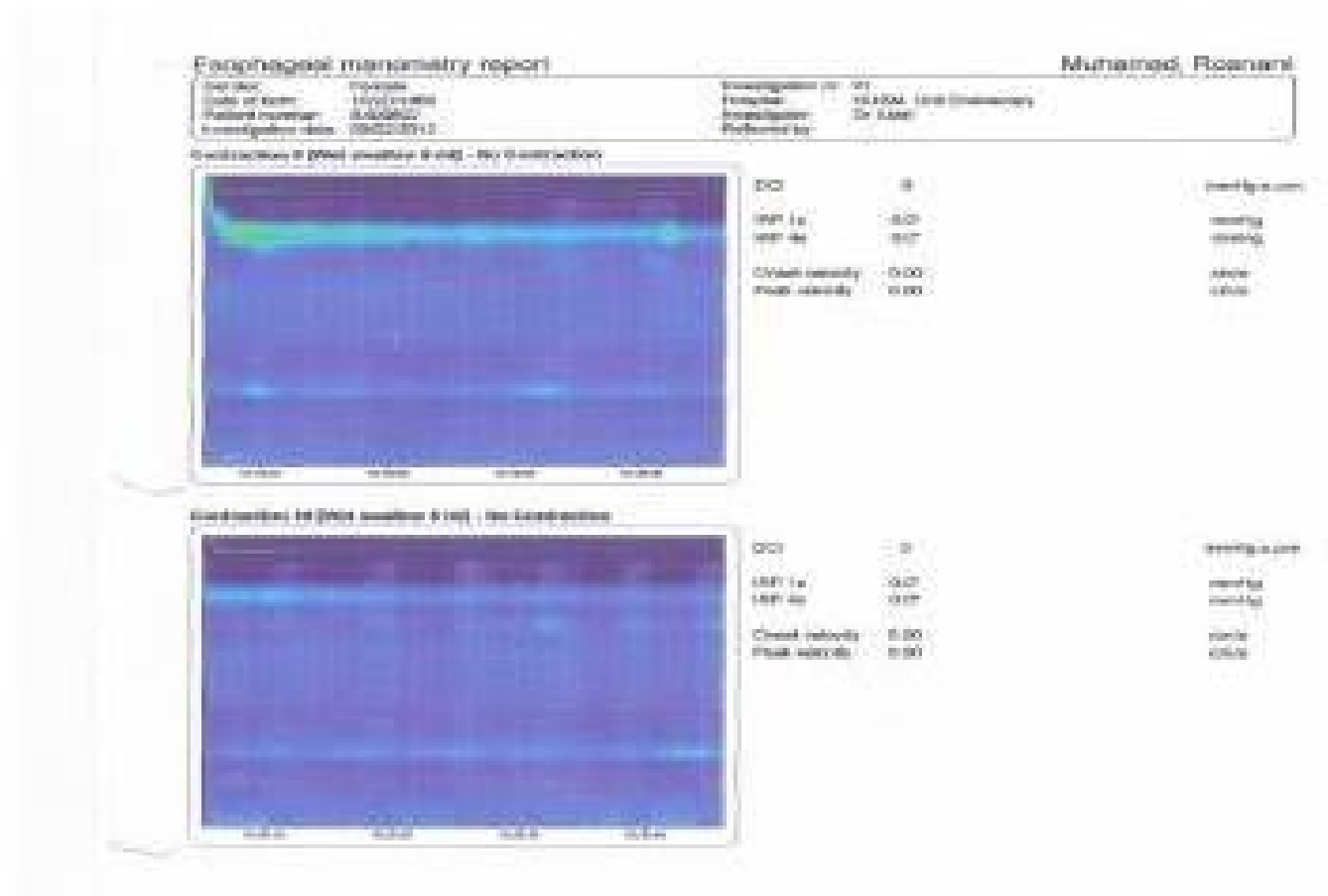

Fig 2(b): Manometry Shows Evidence of No Contraction of Esophagus during Swallowing.

She was initially started with proton pump inhibitor (Pantoprazole). Nystatin suspension was added to treat fungal esophagitis. She was also referred to dietitian for nutritional build up.

She underwent first pneumatic dilatation but without much improvement. For pneumatic dilatation we used 'Wilson-Cook Achalasia Balloon' size $105 \mathrm{Fr}$ at pressure of 40 psig $(2.9$ atm) within 5 minute interval until the balloon easily passed through the stricture region. After the procedure, patient was allowing orally and discharges the next day. The pneumatic dilatation was repeated for 3 occasions without any complications. Second pneumatic dilatation was repeated for 3 occasions without any complications. Second pneumatic dilatation was performed with only improvement of symptoms for 2-3 days. This has subjected her to undergo 3rd pneumatic dilatation, which showed much improvement, as evidenced by able to take orally without vomiting or regurgitation.

Chest radiograph was done post dilatation, which showed no sign of pneumo-mediastinum. However, no follow-up esophageal manometry was done to her in view of the symptoms and her nutrition status was improved.

Barium swallow (Fig. 3) post 3rd pneumatic dilatation still showing bird's beak appearance, which strongly suggest that the symptoms may have relapsed. 


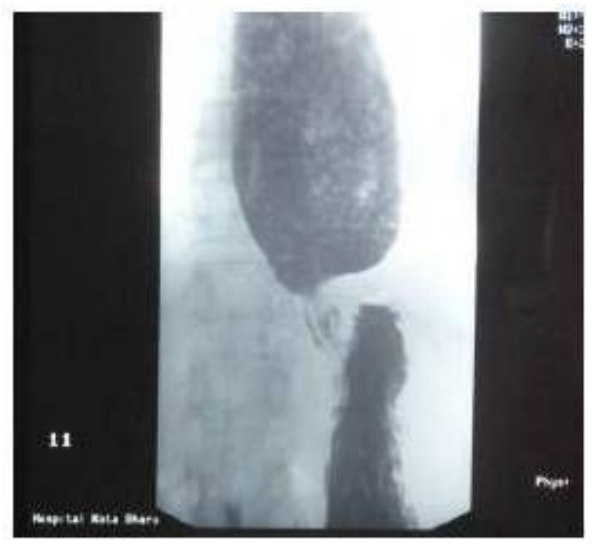

Fig.3: Barium Swallow Showing Dilated Proximal Esophagus with Food Particles and Bird's Beak Appearance.

\section{Discussion}

There are currently four modalities for the treatment of achalasia, which including pharmacological therapy, endoscopic Botulinum toxin A (Botox) injection, pneumatic dilatation and surgical myotomy.

1. Pharmacological therapy, which include isosorbite dinitrate and calcium channel blockers are generally is considered only in patients who are not candidates for surgery or other treatment modalities. However, it can be used for palliating the symptoms while the patient awaits the definitive procedure.

2. Botulinum toxin injection may require repeated sessions to maintain its efficacy. Another drawback is, repeated usage of the Botox will be scleroses the gastroesophageal junction, making surgical myotomy more difficult and prone to result in mucosal injury while doing surgery (John L. Cameron, 2011). The relief of symptoms usually lasts for 3 12 months in $50 \%$ to $60 \%$ of patients after Botox injection.

Endoscopic pneumatic dilatation of the LES may be the most effective treatment for achalasia nonsurgically. However, it can lead to LES incompetence and gastroesophageal reflux in some cases (Wang et al., 2009), and the most severe complication is esophageal perforation.

4. The advantage of surgical myotomy is that disruption of the muscular fibers is accomplished under direct vision. An additional procedure - anti-reflux procedure can be added during the same setting (Kostic et al., 2007b). In the era of advanced technology, the introduction of a laparoscopic approach (minimally invasive) has regained primacy (Rosemurgy et al., 2005). New approaches of using high resolution of manometry in view to determine length of spasm the use of peroral endoscopic myotomy(POEM) as described by (Hubert Louis et al., 2012)

A meta-analysis by Lan Wang et al. (Wang et al., 2009) including five randomized studies compared Botulinum toxin injection with pneumatic dilatation in patients with untreated achalasia, found that there were significant differences in remission rate (pneumatic dilatation $65.8 \%$ versus Botulinum toxin injection 36\%; RR 2.20, 95\% CI 1.51-3.20, P<0.0001) and relapse rate (pneumatic dilatation $16.7 \%$ versus 
Botulinum toxin injection 50\%, RR 0.36, 95\% CI 0.22-0.58) for the two treatment methods. Besides, relapse time of Botulinum toxin injection was shorter than that of pneumatic dilatation after first therapy. It was therefore concluded that pneumatic dilatation is more efficacious than Botulinum toxin injection for treatment of achalasia patients.

In another two randomized studies, Zaninotto et al. (Zaninotto et al., 2004a; Zaninotto et al., 2004b) compared efficacy of Botulinum toxin injection with laparoscopic myotomy in patients with untreated achalasia. These two groups of patients improved and no differences were observed in symptom recurrence during the first 6 months. However, after 1 year of treatment, the remission rate of Botulinum toxin injection rapidly decreased and nearly $50 \%$ of patients were symptomatic again. It was found that laparoscopic myotomy had superior efficacy to Botulinum toxin injection (laparoscopic myotomy $83.3 \%$ versus Botulinum toxin injection 64.9\%, RR 1.28; $95 \%$ CI 1.02-1.59; $\mathrm{P}=0.03$ ).

Two randomized studies (Suarez et al., 2002; Kostic et al., 2007a) compared the efficacy of pneumatic dilation with laparoscopic myotomy in patients with achalasia. The meta-analysis by Lan Wang et al. (Wang et al., 2009) showed that there were significant differences in remission rate (laparoscopic myotomy $95.0 \%$ versus pneumatic dilatation 77.8\%, RR 1.48, 95\% CI 1.16-1.87, $\mathrm{P}=0.001$ ) and relapse rate (laparoscopic myotomy 5.1\% versus pneumatic dilation $35.71 \%$, RR 0.14 , 95\% CI 0.04-0.58, $\mathrm{P}=0.007$ ), indicating that laparoscopic myotomy offered better clinical result than pneumatic dilatation.

A recent meta-analysis by Lan Wang et al. (Wang et al., 2009) suggested that good clinical efficacy combined with low morbidity has established laparoscopic myotomy as a definitive, safe, and effective alternative in the treatment of achalasia. In contrast, pharmacological treatment, Botulinum toxin injection and pneumatic dilatation are temporary and reversible measures.
Laparoscopic myotomy is less invasive than open surgery, but is expensive and the results depend mainly on the surgeon's expertise. The reasons of failure for laparoscopic myotomy were incomplete myotomy (33\%), myotomy fibrosis (27\%), fundoplication disruption (13\%), too tight fundoplication (7\%), and a combination of myotomy fibrosis and incomplete myotomy (20\%) (Wang et al., 2009). In order to reduce the complication rate of laparoscopic myotomy, additional procedure -partial fundoplication is usually performed and becoming popular for treatment of achalasia. Most studies quoted $85 \%$ or more patients have improvement in dysphagia and regurgitation or resolution after laparoscopic Heller myotomy (John L. Cameron, 2011).

Concerning the present case, patient was subjected to pneumatic dilatation, which has shown improvement in the symptoms. However, she needs to be followed up closely as the symptoms will relapse. Nevertheless, we strongly suggest that endoscopic treatment is advisable with frequent followup before surgical treatment commenced. Our center still not started using high resolution manometry and peroral endoscopic myotomy for time being.

\section{Conclusion}

In summary, laparoscopic myotomy is the preferred and recommended method in the management of achalasia. Botulinum toxin injection and pneumatic dilation can offer dysphagia control, but long term relief of dysphagia is significantly less than achieved by surgery. Laparoscopic myotomy with partial fundoplication is superior to other treatment methods because of its excellent efficacy and safety, and also prevent acid exposure in the distal esophagus.

\section{Learning Point}

- Clinical and endoscopic diagnosis of achalasia cardia is important with high index of suspicious. 
- Endoscopic treatment is advisable with frequent follow-up before surgical treatment commenced.

\section{References}

Cameron, J. L. \& Cameron, A. M. (2011). Current Surgical Therapy: Expert Consult, 10th ed.: Elselvier Saunder.

Kostic, S., Johnsson, E., Kjellin, A., Ruth, M., Lonroth, H., Andersson, M. \& Lundell, L. (2007a). "Health Economic Evaluation of Therapeutic Strategies in Patients with Idiopathic Achalasia: Results of a Randomized Trial Comparing Pneumatic Dilatation with Laparoscopic Cardiomyotomy," Surgical Endoscopy, 21(7), 1184-9.

Kostic, S., Kjellin, A., Ruth, M., Lonroth, H., Johnsson, E., Andersson, M. \& Lundell, L. (2007b). "Pneumatic Dilatation or Laparoscopic Cardiomyotomy in the Management of Newly Diagnosed Idiopathic Achalasia. Results of a Randomized Controlled Trial," World Journal of Surgery, 31(3), 470-8.

Louis, H., Covas, A., Coppens, E. \& Devière, J. (2012). "Distal Oesophageal Spasm Treated by Peroral Myotomy," The American Journal of Gastroenterology 2012; 107:1926-1927; 10.1038/ajg.2012.317

Rosemurgy, A., Villadolid, D., Thometz, D., Kalipersad, C., Rakita, S., Albrink, M., Johnson, M. \& Boyce, W. (2005). "Laparoscopic Heller Myotomy Provides Durable Relief from Achalasia and Salvages Failures after Botox or Dilation," Annals of Surgery, 241(5), 725-33; discussion 733-5.

Suarez, J., Mearin, F., Boque, R., Zanon, V., Armengol, J. R., Pradell, J., Bermejo, B. \& Nadal, A. (2002). "Laparoscopic Myotomy vs Endoscopic Dilation in the Treatment of Achalasia," Surgical Endoscopy, 16(1), 75-7.

Vaezi, M. F. \& Richter, J. E. (1998). "Current Therapies for Achalasia: Comparison and Efficacy," Journal of Clinical Gastroenterology, 27(1), 21-35.
Wang, L., Li, Y. M. \& Li, L. (2009). "Metaanalysis of Randomized and Controlled Treatment Trials for Achalasia," Digestive Diseases and Sciences, 54(11), 2303-11.

Zaninotto, G., Annese, V., Costantini, M., Del Genio, A., Costantino, M., Epifani, M., Gatto, G., D'Onofrio, V., Benini, L., Contini, S., Molena, D., Battaglia, G., Tardio, B., Andriulli, A. \& Ancona, E. (2004a). "Randomized Controlled Trial of Botulinum Toxin versus Laparoscopic Heller Myotomy for Esophageal Achalasia," Annals of Surgery, 239(3), 364-70.

Zaninotto, G., Vergadoro, V., Annese, V., Costantini, M., Costantino, M., Molena, D., Rizzetto, C., Epifani, M., Ruol, A., Nicoletti, L. \& Ancona, E. (2004b). "Botulinum Toxin Injection versus Laparoscopic Myotomy for the Treatment of Esophageal Achalasia: Economic Analysis of a Randomized Trial," Surgical Endoscopy, 18(4), 691-5. 